\title{
Age-specific prevalence of cervical human papillomavirus infection and cytological abnormalities in women in Gauteng Province, South Africa
}

\author{
K Richter, ${ }^{1}$ MB ChB, MMed Path (Med Virol), FC Path Med Virol (SA); P Becker, ${ }^{2}$ PhD, MSc; A Horton, ${ }^{3}$ N Dip (Med Tech); \\ G Dreyer, ${ }^{4} \mathrm{PhD}, \mathrm{MCOG}(\mathrm{SA}), \mathrm{MMed}(\mathrm{O \& G})$ \\ ${ }^{1}$ Department of Medical Virology, University of Pretoria, and National Health Laboratory Service, Pretoria, South Africa \\ ${ }^{2}$ Biostatistics Unit, South African Medical Research Council, and Departments of Medical Virology and Gynaecology, University of Pretoria, \\ Pretoria, South Africa \\ ${ }^{3}$ Department of Anatomical Pathology, National Health Laboratory Service, Pretoria, South Africa \\ ${ }^{4}$ Gynaecological Oncology Unit, Pretoria Academic Hospital Complex, University of Pretoria, Pretoria, South Africa
}

Corresponding author: K Richter (karin.richter@up.ac.za)

\begin{abstract}
Background. Women accessing the public health system in Gauteng province, South Africa are largely unscreened for cervical cancer and have a high background prevalence of human immunodeficiency virus (HIV) infection.

Objectives. This cross-sectional study describes the age-specific prevalence of human papillomavirus (HPV) infection and cytological abnormalities among this urban and peri-urban population.

Method. Over the period March 2009 - September 2011, 1524 women attending public sector primary healthcare clinics were invited to participate in a cervical cancer screening study. All participants were screened with conventional cytology and HPV testing undertaken using the HPV linear array genotyping kit (Roche Molecular Systems).

Results. Of 1472 women with valid cytology results, abnormalities were detected in 17.3\% ( $n=255)$, of which 9.1\% ( $n=134)$ were high-grade squamous intraepithelial lesions, and $0.5 \%(n=8)$ suggestive of squamous carcinoma. Of the 1445 women with complete data, the overall and high-risk HPV DNA prevalences were $74.6 \%(n=1078)$ and $54.3 \%(n=784)$, respectively. HPV type 16 and/or 18 were detected in $19.5 \%$ $(n=282)$ of women. Age-specific prevalence of HPV showed a plateau-shaped curve.

Conclusions. The prevalences of HPV infection and abnormal cytology were much higher than previously reported in general populations in South Africa and elsewhere. Higher age-specific prevalence and similar plateau-like age-specific epidemiological curves have previously only been described in studies among HIV-positive women. These findings have implications for planning and development of cervical screening programmes in developing countries with largely unscreened populations with a high background prevalence of HIV.
\end{abstract}

S Afr Med J 2013;103(5):313-317. DOI:10.7196/SAMJ.6514

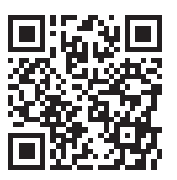

Approximately 6000 new cervical cancer cases are diagnosed annually in South Africa (SA). Accurate contemporary data on cervical cancer incidence have not been available since the 1999 Cancer Registry was published. ${ }^{[1]}$ It is assumed that many cases are still undiagnosed and that more than half of diagnosed patients will die per year. The World Health Organization estimates the agestandardised incidence rate for SA to be 26.6 per 100000 women. ${ }^{[2]}$ The current prevalence of pre-invasive cervical disease in SA is largely unknown. Data from published SA studies suggest important regional differences and an increase in the prevalence of cytological abnormalities when compared with historical data. ${ }^{[3-5]}$ Because of the low sensitivity of cytology, it can be assumed that the true prevalence of pre-invasive disease is underestimated by all available data. A single test of cervical cytology, even if optimal, will probably identify fewer than half of all existing pre-invasive cervical disease when measured against the greater yield obtained at colposcopy and directed biopsy. ${ }^{[6]}$

The identification of high-risk human papillomavirus (hrHPV) types (the cause of cervical cancer) offers the prospect of improving cervical screening programmes through the introduction of hrHPVbased screening tests. Studies from developed countries provide convincing evidence that hrHPV DNA-based screening algorithms are cost-effective and clinically sensitive for the detection of precancerous lesions ${ }^{[7-9]}$ and invasive cervical cancer ${ }^{[10]}$ compared with cytology-based screening in women older than 30. Recently, this finding has also been confirmed in India, a developing country with a low human immunodeficiency virus (HIV) prevalence. ${ }^{[1]}$ Over the last 20 years, the widespread HIV epidemic has increased the overall burden of HPV infection in sub-Saharan Africa. ${ }^{[12]}$ Accurate current knowledge of the genital hrHPV prevalence in developing countries is essential for cost analysis and planning for regionally tailored national prevention and screening programmes. The purpose of this study was to describe the type- and age-specific prevalence of HPV infection and cytological abnormalities among women in Gauteng.

\section{Method}

\section{Study population}

Women attending public sector primary healthcare clinics for routine gynaecological and non-gynaecological primary healthcare-related reasons were invited to participate in a cervical cancer screening study. The study was conducted at 5 clinics in the Tshwane District of Gauteng province that were known to have no cervical cancer screening services in place. The sampling period started in July 2008 and terminated in June 2011. All women with a history of previous or current sexual activity who were able to provide written consent were included in the study. Ethics approval was obtained from the Faculty 
of Health Sciences Research Ethics Committee, University of Pretoria Ethics Board (Protocol no. 210/2008).

\section{Specimen collection and testing}

All participants were screened with conventional cytology (Pap smear) as per national protocol. Cytopathology reports were based on the Bethesda system. ${ }^{[13]}$ Specimens for HPV genotyping were either healthcare worker-collected dry cervical swabs $(N=951)$ or patientcollected tampon specimens $(N=573)$. Tampons were placed in a phosphate buffered saline and $10 \%$ methanol solution directly after collection. DNA extraction was performed in batches on washed cell pellets of the tampon specimens or directly on the dry swab specimens using the DNA Isolation Kit (Roche Molecular Systems, Branchburg, NJ) on the MagNA Pure automated extraction system. HPV genotyping was done using the HPV linear array (LA) genotyping kit (Roche Molecular Systems, Branchburg, NJ). The pool of primers is designed to amplify HPV DNA from 15 high-risk genotypes $(16,18,31,33,35$, $39,45,51,52,56,58,59,68,73$ and 82$), 3$ probable high-risk genotypes (26, 53 and 66) and 19 low/undetermined-risk types $(6,11,40,42,54$, $55,61,62,64,67,69,70,71,72,81,83,84$, IS39 and CP6108). The $\beta$-globin gene was amplified concurrently to assess cellular adequacy, extraction and amplification for each individually processed specimen. Strict procedures were followed to avoid contamination, with negative and positive controls included in each run.

\section{Data and statistical analysis}

HPV genotypes were classified based on the results of Muñoz et al., which regarded 15 types as $\mathrm{hrHPV}(16,18,31,33,35,39,45,51$, $52,56,58,59,68,73$ and 82 ), among which 3 types (26, 53 and 66) are recognised as probable hrHPV for cervical cancer. ${ }^{[14]}$ The 8 most common hrHPV types found in invasive cervical cancer $(16,18,31$, $33,35,45,52,58)$ as described by De Sanjose were classified as the 'top $8{ }^{\text {, }{ }^{[15]}}$ The overall total HPV, type-specific HPV, total hrHPV, 'top 8 hrHPV, and type 16 and/or 18, prevalence and cytology result (normal, atypical squamous cells of undetermined significance (ASCUS)), low-grade squamous intraepithelial lesion (LSIL), highgrade squamous intraepithelial lesion (HSIL) and cervical carcinoma and their 95\% confidence intervals (CIs) were determined for the total study population and per age group. Data were analysed considering study sites as strata associated with the collection method and using sampling weights to account for study design. Since sampling took place without replacement, a finite population correction was also applied to the variance estimator to yield study- design-based CIs for the prevalence of interest. All statistical analyses were performed using Stata software, release 11.0 (StataCorp LP, College Station, Texas, USA).

\section{Results}

\section{Characteristics of the study population}

Of the 1524 women who participated in the study, complete age data and cytology results were available for 1445 and 1472 women respectively. Data excluded from the analysis were the result of no or incomplete specimens received $(n=11)$, no age data available $(n=41)$, cytology not adequate for analysis $(n=11)$ and/or invalid HPV results $(n=20)$. Cytology $(33.0 \%, n=477)$ and HPV genotyping $(4.6 \%, n=66)$ indicated as possibly inadequate because of lack of endocervical cells or low $\beta$-globin gene amplification were included in the analysis. The median age of participating women was 39 years (range $16-83$ ).

\section{HPV DNA prevalence and age distribution}

HPV DNA was detected in $74.6 \%(n=1078)$ of women, with the highest prevalence of $85 \%$ in women younger than 25 years. Overall, hrHPV DNA was present in $54.3 \%(n=784)$ of subjects. The prevalence also peaked in women younger than 25 years and then slightly decreased with age (Table 1 and Figs 1 and 2).

\section{Multiple infections}

Among women with hrHPV infection ( $n=784), 52.7 \%$ had infection with more than one type. Infection with more than one hrHPV type was more common in women younger than 40 years and again showed a rise in women older than 55 years (Fig. 3).

\section{Cervical cytology}

Abnormal cytology was found in $17.3 \%(n=255)$ of samples. ASCUS was reported in $4.7 \%(n=69)$, LSIL in $3.0 \%(n=44)$ and HSIL in $9.1 \%$ $(n=134)$ of patients. Eight patients $(0.5 \%)$ had cytology suggestive of squamous carcinoma (Table 2, Fig. 2). Abnormal cytology was highest in the 30 - 34-year-old age group (25.5\%) with a decline after age 44, matching the gradient of the hrHPV infection curve. HSIL lesions, or worse, were detected in $9.6 \%$ of women overall. These lesions were detected from a young age, with $8.8 \%$ prevalence in women $<25$ years of age, similar to the $9.5 \%$ prevalence in the 25 29 -year age group. The prevalence peaked in the age group $30-34$ years $(16.2 \%)$, with the second-highest prevalence among women aged $40-44(13.6 \%)$.

Table 1. HPV age-specific prevalence percentage (95\% CI) per HPV type or 'pooled' types (see text)

\begin{tabular}{llllllll}
\hline Age group & $N$ & HPV & hrHPV+ & HPV 16+ & HPV 18+ & HPV 16/18+ & HPV 'top 8' \\
\hline$<25$ & 100 & $85.0(75.5-91.4)$ & $66.0(57.6-74.4)$ & $17.0(10.6-23.5)$ & $8.0(3.4-12.6)$ & $24.0(16.6-31.4)$ & $49.0(40.3-57.7)$ \\
$25-29$ & 186 & $79.6(73.1-85.1)$ & $62.9(56.7-69.1)$ & $15.1(10.5-19.6)$ & $9.7(5.9-13.4)$ & $22.0(16.8-27.3)$ & $52.2(45.7-58.6)$ \\
$30-34$ & 233 & $79.4(73.6-84.4)$ & $61.8(56.5-67.1)$ & $18.0(13.7-22.4)$ & $9.9(6.5-13.2)$ & $24.5(19.6-29.3)$ & $51.1(45.5-56.6)$ \\
$35-39$ & 215 & $72.6(66.1-78.4)$ & $59.5(53.9-65.2)$ & $14.0(9.9-18.1)$ & $8.4(5.2-11.6)$ & $21.4(16.6-26.2)$ & $50.7(45.0-56.4)$ \\
$40-44$ & 206 & $77.2(70.8-82.7)$ & $55.8(49.9-61.8)$ & $14.1(9.9-18.2)$ & $6.3(3.4-9.2)$ & $18.5(13.9-23.0)$ & $42.7(36.8-48.6)$ \\
$45-49$ & 180 & $67.8(60.4-74.5)$ & $44.4(38.0-50.9)$ & $10.6(6.6-14.5)$ & $6.7(3.4-9.9)$ & $15.6(10.9-20.2)$ & $34.4(28.3-40.6)$ \\
$50-54$ & 146 & $64.4(56.0-72.1)$ & $40.4(33.4-47.5)$ & $10.3(6.0-14.6)$ & $5.5(2.3-8.6)$ & $13.7(8.9-18.5)$ & $27.4(21.0-33.8)$ \\
$\geq 55$ & 179 & $72.1(64.9-78.5)$ & $41.9(35.7-48.1)$ & $8.4(4.8-12.0)$ & $8.4(4.8-12.0)$ & $15.6(10.9-20.3)$ & $36.3(30.3-42.4)$ \\
Total & 1445 & $74.6(69.5-79.1)$ & $54.3(53.0-56.5)$ & $13.5(12.0-15.0)$ & $8.0(6.7-9.2)$ & $19.5(17.7-21.3)$ & $43.5(41.3-45.8)$ \\
hrHPV = high-risk human papillomavirus. & & & &
\end{tabular}




\section{RESEARCH}

Table 2. Cytological abnormality age-specific prevalence percentage (95\% CI)

\begin{tabular}{lllllll}
\hline Age group & $\boldsymbol{N}$ & Cytology abnormal & ASCUS & LSIL & HSIL & Cervical carcinoma \\
\hline$<25$ & 102 & $16.7(10.0-25.3)$ & $2.0(0.2-6.9)$ & $5.9(2.2-12.4)$ & $8.8(4.1-16.1)$ & $0.0(0.0-0.0)$ \\
$25-29$ & 189 & $19.6(14.2-26.0)$ & $5.8(2.9-10.2)$ & $4.2(1.8-8.2)$ & $9.0(5.3-14.0)$ & $0.5(0.0-2.9)$ \\
$30-34$ & 235 & $25.5(20.1-31.6)$ & $5.1(2.7-8.8)$ & $4.3(2.1-7.7)$ & $15.7(11.3-21.0)$ & $0.4(0.0-2.4)$ \\
$35-39$ & 220 & $19.1(14.1-24.9)$ & $5.0(2.5-8.8)$ & $2.3(0.1-5.2)$ & $11.8(7.9-16.8)$ & $0.0(0.0-1.7)$ \\
$40-44$ & 214 & $22.0(16.6-28.1)$ & $5.6(2.9-9.6)$ & $2.8(1.0-6.0)$ & $12.6(8.5-17.8)$ & $0.9(0.1-3.4)$ \\
$45-49$ & 182 & $10.4(6.4-15.8)$ & $5.0(2.3-9.2)$ & $1.7(0.3-4.7)$ & $1.7(0.3-4.7)$ & $2.2(0.6-5.5)$ \\
$50-54$ & 149 & $11.4(6.8-17.6)$ & $2.7(0.7-6.7)$ & $2.7(0.7-6.7)$ & $6.0(2.8-11.2)$ & $0.0(0.0-2.5)$ \\
$\geq 55$ & 181 & $8.8(5.1-14.0)$ & $4.4(1.9-8.5)$ & $1.1(0.1-3.9)$ & $3.3(1.2-7.1)$ & $0.0(0.0-2.0)$ \\
Total & 1472 & $17.3(12.6-23.4)$ & $4.7(3.9-5.7)$ & $3.0(2.0-4.4)$ & $9.1(5.6-14.5)$ & $0.5(0.2-1.7)$ \\
ASCUS = atypical squamous cells of undetermined significance; LSIL = low-grade squamous intraepithelial lesion; HSIL = high-grade squamous intraepithelial lesion.
\end{tabular}

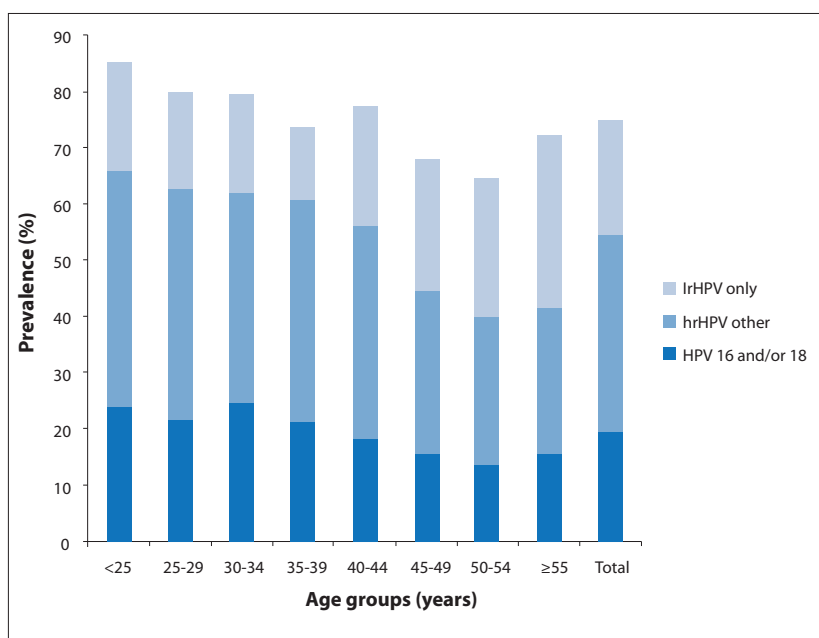

Fig. 1. Cumulative age-specific prevalence of any HPV DNA divided into $H P V 16$ and/or 18 positive, hrHPV other than type 16 or 18 positive, and only lrHPV positive.

\section{Discussion}

\section{HPV DNA prevalence and age distribution}

The prevalence of cervical HPV infection, overall and by age, varies by continent, country, region and population subgroup. Reported prevalence is also affected by sampling method and the sensitivity of the chosen assay. The highest prevalence is reported in studies conducted in populations in sub-Saharan Africa and in HIV-positive cohorts. A study among women from urban and rural Tanzania demonstrated an hrHPV prevalence of $20.1 \%$ using the hybrid capture 2 (HC2) test (Qiagen) ${ }^{[16]}$ Another population-based study from rural Nigeria used a modified MY09-MY11 polymerase chain reaction (PCR)-based method and found an hrHPV prevalence of $14.7 \% .{ }^{[17]} \mathrm{A}$ smaller study $(N=153)$ from our region reported an overall HPV prevalence of $58 \%$ using the LA. ${ }^{[18]}$ Snijders et al. recommend that to accurately study HPV epidemiology, impact of HPV infection and monitor the efficacy of HPV vaccination programmes, the test with the highest analytical sensitivity possible must be used. ${ }^{[19]}$ This shift toward highly sensitive PCR assays has affected findings in recent epidemiological studies, showing that HPV infections are more widespread than had previously been recognised. ${ }^{[20]}$ In the current study, the highly analytically sensitive HPV LA was used. The overall HPV and hrHPV prevalence of 74.6\% and $54.3 \%$ represents the highest prevalence ever reported in a sample of the general population.

HPV prevalence is described as peaking in women $<25$ years old, with a rapid fall in their 30s and $40 \mathrm{~s}^{[2]}$ Inconsistent trends in HPV prevalence

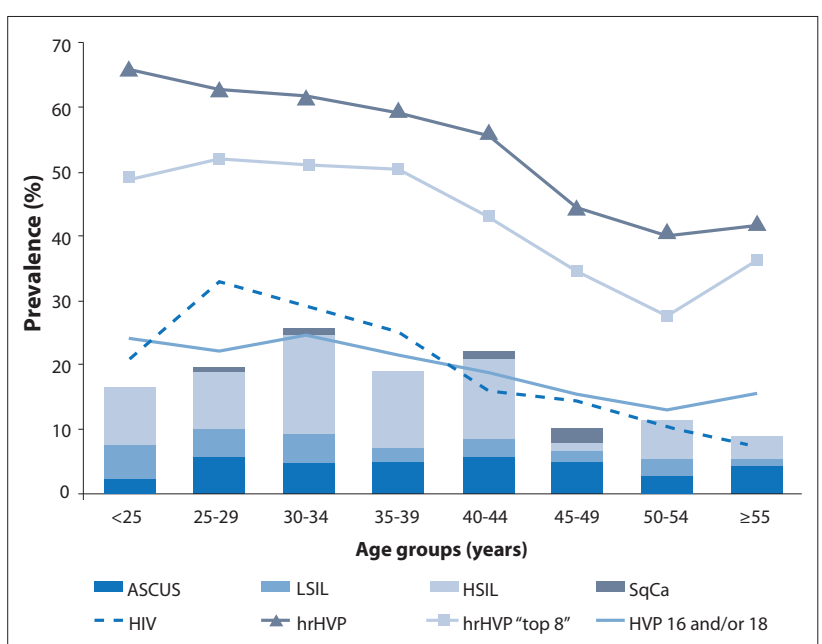

Fig. 2. Age-specific prevalence of $h r H P V$, 'top 8 ' cancer-causing $h r H P V$, HPV type 16 and/or 18 positive, cytological abnormalities ${ }^{\ddagger}$ and estimated background HIV prevalence.

${ }^{*} H P V$ DNA positive for one or more of 15 oncogenic HPV types.

${ }^{\dagger}$ The eight most common hrHPV types found in invasive cervical cancer $(16,18,31,33,35,45,52,58) .{ }^{[15]}$ ${ }^{\ddagger}$ Classification by severity: atypical squamous cells of undetermined significance (ASCUS), low-grade
squamous intraepithelial lesion (LSIL), high-grade squamous intraepithelial lesion (HSIL), cervical squamous carcinoma $(S q C a)$.

${ }^{5}$ Most recent population estimates. ${ }^{[27]}$

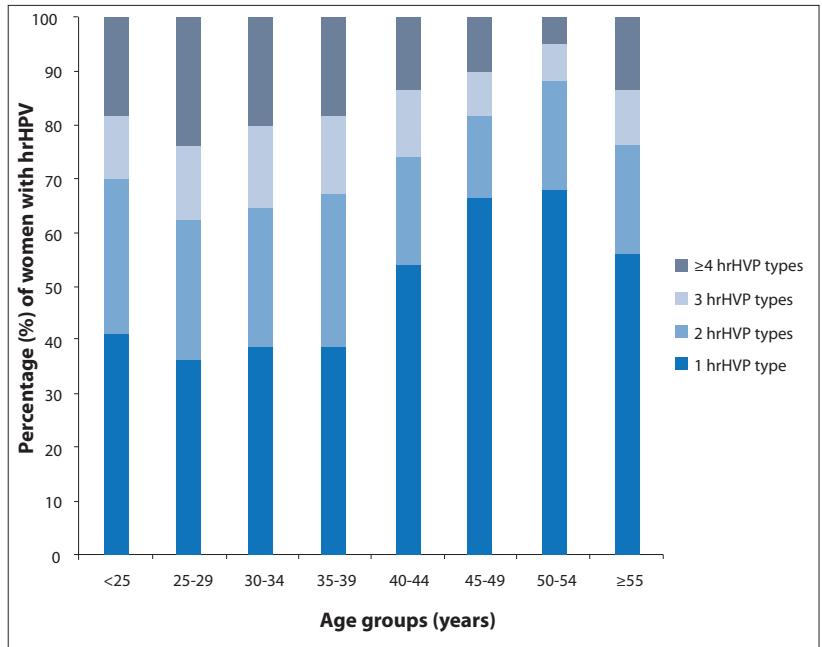

Fig. 3. Age-specific prevalence of single v. multiple high-risk HPV types (hrHPV) as a percentage of women with hrHPV infections. 
are noted in older women, with most studies reporting a decrease of HPV infection in older ages while others document an increase or second peak. ${ }^{[2,22]}$ Elevated HPV prevalence across all age groups, with flat or plateau-like age curves, has been observed in the lowest-income areas of Asia and Nigeria along with high or very high incidence of cervical cancer and mortality. This finding has also been documented in communities with no or inadequate screening programmes ${ }^{[2,23]}$ and among HIV-positive women living on different continents. ${ }^{[2]}$

The high HPV prevalence and plateau-like age curve observed in this cohort could reflect several factors. These include sexual behavioural patterns, the largely unscreened and therefore untreated status of the female population owing to the inadequate implementation of cervical cancer screening programmes, ${ }^{[2,25]}$ as well as the high background HIV prevalence (Fig. 2) and associated immune compromise in SA women. From 2009 to 2011, it was estimated that $19.1-19.4 \%$ of SA women aged 15 - 49 years were HIV positive. ${ }^{[2]]}$ The national HIV prevalence survey, published in 2008, found that HIV prevalence peaks in females aged $25-29$ years at $32.7 \%$, and ranges from $21.1 \%$ in 20 - 24-year-olds, to $29.1 \%$ in the $30-34$ age group, $24.8 \%$ in the $35-39$ group, $16.3 \%$ in the 40 - 44 group, and $14.1 \%$ in the $45-49$ group. ${ }^{[27]}$ Subsequent antenatal survey trend data from 2007 to 2010 showed that HIV prevalence was increasing among women aged 30 and older, ${ }^{[28]}$ with a shift of the peak HIV prevalence to the age category $30-34$ years. ${ }^{[26]}$ Several studies have demonstrated a significantly higher prevalence of cervical cancer pre-cancerous lesions and HPV infection in HIV-infected women than among HIV-uninfected women ${ }^{[12,16,629-31]}$ HIV-infected women also have a higher frequency of infection with multiple HPV types, ${ }^{[3,1,32]}$ and persistence or reactivation of infections is more likely. ${ }^{[2,33]}$ Increased and accelerated progression rates to cervical cancer also lead to a younger mean age at diagnosis of pre-invasive and invasive disease. ${ }^{[34]}$

It is crucial that HIV testing be promoted among women being screened for cervical cancer. Importantly, cervical cancer screening should become an integral part of the chronic care of HIV-positive women. It is known that the risk of both HPV infection and cervical intraepithelial neoplasia increases with increasing degrees of immunosuppression. In SA, the general uptake of HIV testing is low, with late initiation of highly active antiretroviral therapy (HAART) at an average CD 4 count of 87 cells $/ \mu$. In 2010 , only $56 \%$ of those eligible for HAART in SA were receiving it. ${ }^{[28]}$

\section{Multiple infection rate}

Co-infection with multiple HPV types can be seen as an independent indicator of risk. ${ }^{[20]}$ The high prevalence of multiple HPV infections and very high count of types in single patients probably reflects the combination of using a highly sensitive PCR assay and several risk factors in this population. The latter include: a high frequency of unprotected intercourse with multiple sexual partners in a population with a high background prevalence of HPV infection; a relative state of immune compromise in a large subset of the population owing to advanced and sub-optimally treated HIV infection or nutritional status; and genetic differences in host susceptibility. ${ }^{[20,35]}$

\section{Cytology}

Previous local data from 2006 showed an abnormal cytology rate of $7.3 \%$ in the Western Cape and $10 \%$ in Gauteng. ${ }^{[25]}$ The higher abnormality rate of $17.3 \%$ detected in this study population is expected, when considering the high HPV infection rate. Traditionally, highgrade lesions are typically diagnosed in women 25 - 35 years old, while invasive cancer is more commonly diagnosed after age 40 years, typically $8-13$ years after diagnosis of a high-grade lesion. Of particular concern is that HSILs were seen in more than $8 \%$ of women $<25$ years old.

\section{Limitations}

Full genotyping assays are not advocated as screening assays because of their very high analytical sensitivity and low clinical specificity, their high cost, and labour-intensive laboratory methodology. Studies among women with cytological abnormalities have shown HC2 to have lower HPV positivity compared with PCR assays such as the LA. ${ }^{[36,37]}$ The observed high HPV prevalence can not, however, be attributed to the sensitivity of the assay alone, as reflected by the concurrent high prevalence of cytological abnormalities and the pattern of multiple and single HPV infections that appears to mirror the background HIV prevalence per age groups (Figs 2 and 3). In view of the well-known large regional and population differences in HPV epidemiology, the results of this study cannot be generalised to the whole region or to the country.

\section{Implications for primary prevention}

The high sensitivity of the LA assay is considered of significant value as an epidemiological tool for prevalence studies of HPV infections as well as for pre- and post-prophylactic vaccine intervention. ${ }^{[3]}$ These data predict that prophylactic vaccination of females prior to their sexual debut in this and similar populations could potentially prevent a large proportion of cervical HPV infections attributed to HPV 16 and 18. Catch-up vaccination will be less beneficial and cost-effective, as at least $20 \%$ of women $<25$ years old are already exposed to HPV 16 and/ or 18 (Fig. 2). HAART can effectively increase cell-mediated immunity among sexually active people, which will reduce the burden of HPV infection in the population. Patterns of sexual behaviour that influence the epidemiology of HPV infection are more difficult to change.

\section{Implications for secondary prevention}

If women in developing regions are screened, it is usually only once or twice in a lifetime. The South African National Department of Health has a target of 70\% cervical cancer screening coverage with cytology repeated at 10-year intervals. In 2006, Denny reported that less than $10 \%$ of the targeted female population were actually screened. In some provinces, the coverage was as low as $2 \% \cdot{ }^{[25]}$ It is of utmost importance to maximise sensitivity when a long screening interval is used and coverage is poor. ${ }^{[36]}$ These data predict that an effective screening programme with a highly sensitive hrHPV DNA assay, in this and similar populations, will place an enormous burden on the healthcare system owing to the increase in the number of women requiring triage, follow-up and, ultimately, treatment. These results show that more than half of women will need further healthcare intervention if an hrHPV test-and-treat policy were to be introduced in women $>30$ years old. Highly sensitive non-discriminatory HPV DNA tests are therefore unsuitable for primary screening without further triage in low-resource settings. The ideal screening test should balance sensitivity with high clinical specificity or positive predictive value for disease.

To achieve $70 \%$ coverage of the population in SA, over 5 million women $>30$ years old would need to be screened every 10 years. ${ }^{[25]}$ HPV genotyping or pooled partial genotyping of the 'highest risk' or 'higher risk' cervical cancer-causing HPV types will increase specificity while maintaining the sensitivity of the initial round of screening. The 'highest risk' viruses can be defined as HPV 16 and/ or 18, while the most prevalent four, six or eight HPV types found in cervical cancer can be chosen as the 'higher risk' types for screening purposes. Depending on the choice, the trade-off between sensitivity and specificity can be made according to cost-effectiveness. With $100 \%$ coverage, screening for the top four types should prevent an estimated $80 \%$ of cancerous lesions, while screening for the 'highest risk' types only can be projected to prevent $<70 \%$ in this context. ${ }^{[3]}$ 
Data from this study can assist in predicting the possible outcomes of different screening test choices in this context. The age-specific prevalence curve shows that approximately 1 in every 4 women aged $25-35$, or 1 in every 5 women aged $35-45$, will need treatment if positivity for HPV 16 or 18 is used as a stratification and treatment criterion. These percentages are comparable to the prevalence of cytological abnormalities in the same age groups. Screening for the presence of the 'top 8 ' HPV types will identify approximately $50 \%$ of women between 25 and 40 years and approximately $40 \%$ of women aged $>40$ who are at higher risk (Figs 1 and 2).

\section{Conclusion}

This unscreened female population, having a high background prevalence of sub-optimally treated HIV, is typical of many populations in the developing world. HPV infection, co-infection with several hrHPV types and abnormalities on cytology were more prevalent than previously appreciated. The plateau-like curve seen among age groups $<45$ years is unique. All these phenomena probably reflect a combination of factors. Follow-up data are needed to clarify to what extent the high HPV prevalence in middle- and older-aged women is related to the persistence or reactivation of old infections, acquisition of new infections, or cohort effects.

The increased HPV prevalence determined in this sample of the SA population underscores the urgent need for improved cervical cancer screening and prevention programmes to prevent a marked increase in the incidence of cervical cancer in the next decade. These data provide an indication of the $40-50 \%$ of women who would require followup if primary HPV screening were introduced. It also indicates the magnitude of possible benefit were prophylactic HPV vaccination to be introduced. Future evaluations of the performance of new clinically sensitive and specific HPV screening tests are required to design practical and realistic screening programmes for SA.

Acknowledgements. We thank Judy Hasset for data capturing; Aubrey Morulane, Corrie de Villiers and Nanette Heunis for laboratory support; Edwin Mnisi and Alta Mongwe for site co-ordination; and Rika Byleveldt for administrative support. This study was funded by a grant from the Cancer Association of South Africa (CANSA).

\section{References}

1. Mqoqi N, Kellett P, Sitas F, Jula M. Incidence of Histologically Diagnosed Cancer in South Africa 19981999. Johannesburg: National Cancer Registry of South Africa, National Health Laboratory Service, 2004. 2. WHO/ICO Information Centre on HPV and Cervical Cancer. Human papillomavirus and related cancers in South Africa. Summary Report 2010. http://who.int/hpvcentre (accessed 15 November 2012).

3. Fonn S, Bloch B, Mabina M, et al. Prevalence of pre-cancerous lesions and cervical cancer in South Africa -a multicentre study. S Afr Med J 2002;92:148-156.

4. Allan BR, Marais DJ, Denny L, et al. The agreement between cervical abnormalities identified by cytology and detection of high-risk types of human papillomavirus. S Afr Med J 2006;96:1186-1190.

. Taylor S, Kuhn L, Dupree W, et al. Direct comparison of liquid-based and conventional cytology in a South African screening trial. Int J Cancer 2006;1 18:957-962. [http://dx.doi.org/10.1002/ijc.21434]

6. Castle PE, Stoler MH, Wright TC Jr, et al. Performance of carcinogenic human papillomavirus (HPV) testing and HPV 16 or HPV 18 genotyping for cervical cancer screening of women aged 25 years and older A subanalysis of the ATHENA study. Lancet Oncol 2011;12:880-890.

Bulkmans N, Berkhof J, Rozendaal L, et al. Human papillomavirus DNA testing for the detection of cervical intraepithelial neoplasia grade 3 and cancer: 5-year follow-up of a randomised controlled implementation trial. Lancet 2007;370:1764-1772. [http://dx.doi.org/10.1016/S0140-6736(07)61450-0]
Naucler P, Ryd W, Törnberg S, et al. Human papillomavirus and Papanicolau tests to screen for cervical cancer. N Engl J Med 2007;357:1589-1597. [http://dx.doi.org/10.1056/NEJMoa073204]

Kitchener HC, Almonte M, Thomson C, et al. HPV testing in combination with liquid-based cytology in primary cervical screening (ARTISTIC): A randomised controlled trial. Lancet Oncol 2009;10:672-682. http://dx.doi.org/10.1016/S1470-2045(09)70156-1]

10. Ronco G, Giorgi-Rossi P, Carozzi F, et al. Efficacy of human papillomavirus testing for the detection of invasive cervical cancers and cervical intraepithelial neoplasia: A randomised controlled trial. Lancet Onco 2010;11:249-257. [http://dx.doi.org/10.1016/S1470-2045(09)70360-2]

11. Sankaranarayanan R, Nene BM, Shastri SS, et al. HPV screening for cervical cancer in rural India. N Engl Med 2009;360:1385-1394. [http://dx.doi.org/10.1056/NEJMoa0808516]

12. Clifford GM, Franceschi S. HPV in sub-Saharan Africa (editorial). Papillomavirus Rep 2005;16:322-326 [http://dx.doi.org/10.1179/095741905X49089]

13. Solomon D, Nayar R, eds. The Bethesda System for Reporting Cervical Cytology. 2nd ed. New York: Springer Verlag, 2004:191.

14. Muñoz N, Bosch FX, De Sanjosé S, et al. Epidemiologic classification of human papillomavirus types associated with cervical cancer. N Engl J Med 2003;348:518-527. [http://dx.doi.org/10.1056/NEJMoa021641]

15. De Sanjose S, Quint WGV, Alemany L, et al. Human papillomavirus genotype attribution in invasive cervical cancer: A retrospective cross-sectional worldwide study. Lancet Oncol 2010;11:1048-1056. [http:// dx.doi.org/10.1016/S1470-2045(10)70230-8

16. Dartell M, Rasch V, Kahesa C, et al. Human papillomavirus prevalence and type distribution in $3603 \mathrm{HIV}$ positive and HIV-negative women in the general population of Tanzania: The PROTECT study. Sex Transm Dis 2012;39(3):201-208. [http://dx.doi.org/10.1097/OLQ.0b013e31823b50ad]

17. Gage JC, Ajenifuja KO, Wentzensen NA, et al. The age-specific prevalence of human papillomavirus and risk of cytologic abnormalities in rural Nigeria: Implications for screen-and-treat strategies. Int J Cancer 2012;130:2211-2217. [http://dx.doi.org/10.1002/ijc.26211]

18. Said HM, Ahmed K, Burnett R, et al. Human papillomavirus genotypes in women with squamous intraepithelial lesions and normal cervixes participating in a community-based microbicide study in Pretoria, South Africa. J Clin Virol 2009;44:318-321. [http://dx.doi.org/10.1016/j.jcv.2009.02.001]

19. Snijders PJ, Van den Brule AJ, Meijer CJ. The clinical relevance of human papillomavirus testing: relationship between analytical and clinical sensitivity. J Pathol 2003;201:1-6. [http://dx.doi.org/10.1002/path.1433]

20. Plummer M, Vaccarella S, Franceschi S. Multiple human papillomavirus infections: The exception or the rule? [editorial]. J Infect Dis 2011;203:891-893. [http://dx.doi.org/10.1093/infdis/jiq146]

21. Smith JS, Melendy A, Rana RK, Pimenta JM. Age-specific prevalence of infection with human papillomavirus in females: A global review. J Adolescent Health 2008;43:S5-25. [http://dx.doi.org/10.1016/j. jadohealth.2008.07.009]

22. Franceschi S, Herrero R, Clifford GM, et al. Variations in the age-specific curves of human papillomaviru prevalence in women worldwide. Int J Cancer 2006;119:2677-2684. [http://dx.doi.org/10.1002/ijc.22241]

23. Gage JC, Partridge EE, Rausa A, et al. Comparative performance of human papillomavirus DNA testing using novel sample collection methods. J Clin Microbiol 2011;49(12):4185-4189. [http://dx.doi.org/10.1128/ JCM.01254-11]

24. Cronje HS, Beyer E. Screening for cervical cancer in an African setting. Int J Gynaecol Obstet 2007;98: 168171. [http://dx.doi.org/10.1016/ji.jigo.2007.05.005]

5. Denny L. Prevention of cervical cancer. Reprod Health Matters 2008;16:18-31. [http://dx.doi.org/10.1016/j. ijgo.2007.05.005]

26. Statistics South Africa. Mid-year Population Estimates 2011. http://www.statssa.gov.za/publications/P0302/ P03022011.pdf (accessed 15 November 2012)

27. Shisana O, Rehle T, Simbayi LC, et al. South African National HIV Prevalence, Incidence, Behaviour and Communication Survey 2008: A Turning Tide among Teenagers? Cape Town: HSRC Press, 2009:98.

28. UNAIDS. Country Progress Report on the Declaration of Commitment on HIV/AIDS - 2010. http://data. unaids.org/pub/report/2010/southafrica_2010_country_progress_report_en.pdf (accessed 15 November 2012). De Vuyst H, Lillo F, Broutet N, Smith JS. HIV, human papillomavirus, and cervical neoplasia and cancer in the era of highly active antiretroviral therapy. Eur J Cancer Prev 2008;17:545-554. [http://dx.doi. org/10.1097/CEJ.0b013e3282f75eal]

30. Palefsky J. Human papillomavirus-related disease in people with HIV. Curr Opin HIV AIDS 2009:4:52-56. [http://dx.doi.org/10.1097/COH.0b013e32831a7246]

31. Levi JE, Kleter B, Quint WGV, et al. High prevalence of human papillomavirus (HPV) infections and high frequency of multiple HPV genotypes in human immunodeficiency virus-infected women in Brazil. I Clin frequency of multiple HPV genotypes in human immunodeficiency virus-infected wom
Microbiol 2002;40(9):3341-3345. [http://dx.doi.org/10.1128/JCM.40.9.3341-3345.2002]

32. Strickler HD, Burk RD, Fazzari M, et al. Natural history and possible reactivation of human papillomavirus . Strickler HD, Burk RD, Fazzari M, et al. Natural history and possible reactivation of human papillomavirus
in human immunodeficiency virus-positive women. J Natl Cancer Inst 2005;97:577-586. [http://dx.doi. in human immunodeficie
org/10.1093/jnci/dji073]

33. Theiler RN, Farr SL, Karon JM, et al. High-risk human papillomavirus reactivation in human Theiler RN, Farr SL, Karon JM, et al. High-risk human papillomavirus reactivation in human
immunodeficiency virus-infected women: Risk factors for cervical viral shedding. Obstet Gynecol immunodeficiency $2010: 115 \cdot 1150-1158$

4. Lomalisa P, Smith T, Guidozzi F. Human immunodeficiency virus infection and invasive cervical cancer in South Africa. Gynecol Oncol 2000;77:460-463. [http://dx.doi.org/10.1006/gyno.2000.5775]

35. Chaturvedi AK, Katki HA, Hildesheim A, et al. Human papillomavirus infection with multiple types: . Chaturvedi AK, Katki HA, Hildesheim A, et al. Human papillomavirus infection with multiple types Pattern of coinfection and risk of cervical disease. J Infect Dis 2011;203:910-920. [http://dx.doi.org/10.1093
infdis/jii139]

Meijer CJ, Snijders PJ, Castle PE. Clinical utility of HPV genotyping. Gynecol Oncol 2006;103:12-17. [http:/ dx.doi.org/10.1016/j.ygyno.2006.07.031]

37. Stevens MP, Garland SM, Rudland E, et al. Comparison of the Digene Hybrid Capture 2 assay and Roche Amplicor and Linear Array human papillomavirus (HPV) tests in detecting high-risk HPV genotypes in specimens from women with previous abnormal Pap smear results. J Clin Microbiol 2007;45:2130-2137.

38. Guan P, Howell-Jones R, Li N, et al. Human papillomavirus types in 115,789 HPV-positive women: A metaanalysis from cervical infection to cancer. Int I Cancer 2012;131(10):2349-2359. [http://dx.doi.org/10.1002/ ijc.27485]

Accepted 16 January 2013

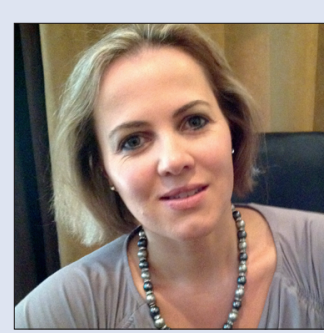

Karin Richter is a clinical virologist, consultant pathologist and senior lecturer at the Department of Medical Virology, University of Pretoria and National Health Laboratory Service (NHLS), Tshwane Academic Division. Her research interests include preventative medicine, HIV and women's health issues. Current research projects, in association with the Gynaecological Oncology Unit, Pretoria Academic Hospital Complex, University of Pretoria, focus on human papillomavirus (HPV) epidemiology and improvement of cervical cancer screening and vaccination protocols in South Africa and other developing countries by incorporating new HPV-based tests and patient self-sampling.

${ }^{\ddagger}$ Richter K, Becker P, Horton A, Dreyer G. Age-specific prevalence of cervical human papillomavirus infection and cytological abnormalities in women in Gauteng province, South Africa. S Afr Med J 2013;103(5):313-317. [http://dx.doi.org/10.7196/SAMJ.6514] 